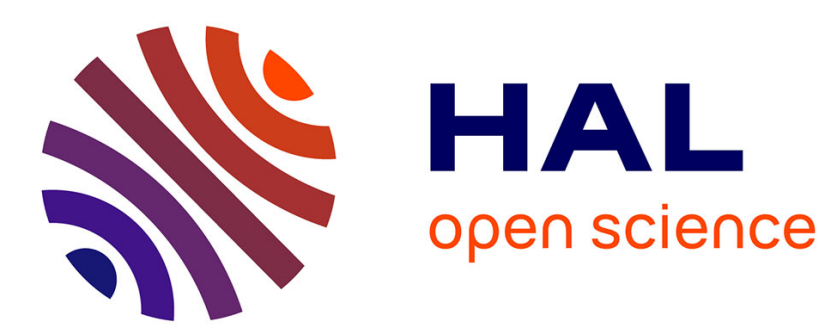

\title{
Decay of Turbulence in Pipe Flow
}

\author{
Jorge Peixinho, Tom Mullin
}

\section{To cite this version:}

Jorge Peixinho, Tom Mullin. Decay of Turbulence in Pipe Flow. Physical Review Letters, 2006, 96 (94501), 10.1103/PhysRevLett.96.094501 . hal-01811237

\section{HAL Id: hal-01811237 https://hal.science/hal-01811237}

Submitted on 8 Jun 2018

HAL is a multi-disciplinary open access archive for the deposit and dissemination of scientific research documents, whether they are published or not. The documents may come from teaching and research institutions in France or abroad, or from public or private research centers.
L'archive ouverte pluridisciplinaire HAL, est destinée au dépôt et à la diffusion de documents scientifiques de niveau recherche, publiés ou non, émanant des établissements d'enseignement et de recherche français ou étrangers, des laboratoires publics ou privés. 


\title{
Decay of Turbulence in Pipe Flow
}

\author{
J. Peixinho and T. Mullin \\ School of Physics and Astronomy, The University of Manchester, Manchester M13 9PL, United Kingdom
} (Received 15 November 2005; published 8 March 2006)

\begin{abstract}
A novel experiment has been devised which provides direct evidence for critical point behavior in the longstanding problem of the transition to turbulence in a pipe. The novelty lies in the quenching of turbulence by reducing the Reynolds number and observing the decay of disordered motion. Divergence of the time scales implies underlying deterministic dynamics which are analogous to those found in boundary crises in dynamical systems. A modulated wave packet emerges from the long term transients and this coherent state provides evidence for connections with recent theoretical developments.
\end{abstract}

DOI: 10.1103/PhysRevLett.96.094501

PACS numbers: 47.20. $-\mathrm{k}, 47.27 .-\mathrm{i}, 47.60 .+\mathrm{i}$

The enigma of why the fluid motion in a pipe becomes turbulent as the flow rate increases has puzzled the physics community for more than a century [1,2]. All evidence suggests that the flow is linearly stable [3] for all Re. (Here $\operatorname{Re}=U D / \nu$ where $U$ is the mean speed, $D$ is the diameter of the pipe, and $\nu$ is the kinematic viscosity of the fluid.) In practice, however, pipe flows are observed to be turbulent even at modest flow rates. The transition to turbulence occurs above a definite amplitude of an injected perturbation and is catastrophic [4]. It is difficult to isolate the effective part of the perturbation, so that the mechanism for transition remains aloof. Therefore, we have devised a novel experiment to investigate the reverse transition, i.e., the change from turbulent to laminar flow. The flow can now be considered as a dynamical system where the turbulent attractor loses stability at a crisis as a parameter is changed $[5,6]$. Exponential decay of the disordered motion is found when the Reynolds number is reduced and, moreover, the observed divergence in the time scales indicates an underlying critical event. Interestingly, the long term decaying transients contain wavelike structures which point the way to direct connections with modern theoretical developments $[7,8]$.

The size of the disturbance required to cause transition has been shown to scale as $\mathrm{Re}^{-1}$ [9]. This relationship cannot hold for small values of $\mathrm{Re}$ and it was recently shown that there is a sharp cutoff [10] at $\operatorname{Re} \sim 1800$, i.e., below this value sustained turbulent flow cannot be created by injecting large amplitude disturbances. Further, obtaining clear estimates of this lower limit using significant perturbations produces large scatter in the data since it is known that the flow is sensitive to background noise [1]. The transition process which takes the flow from the laminar to turbulent states is catastrophic [4] and it is thus difficult to obtain insight into the detailed mechanisms involved in the creation of turbulence from onset experiments. Furthermore, the final state which evolves out of the transition process has the form of a localized patch of disordered motion [11,12] in the Re range 1800-3000. Their spatial extent is constant for $\operatorname{Re}<2223$ [13] and in this Re range they have been called "equilibrium puffs"
[11] since they do not change their form. Puffs travel at a well defined speed of $\simeq 0.9 U$, are approximately 20 pipe diameters long, and comprise a block of disordered flow with a weak decaying wave at the front and a sharp rear interface. They are structured [11] and, recently, more details of the structure [14] have been revealed. Crosssectional views contain azimuthal features which are similar to traveling waves found in recent theoretical work $[7,8]$.

Our strategy was to generate a turbulent puff and observe its decay back to Poiseuille flow as Re was reduced in a well-controlled manner. The puff can be considered as a natural state or attractor for the system. The objective was to see if the reverse transition was less abrupt, which in turn may reveal more definite evidence for traveling wave states. The decay of turbulence in pipe flows has been investigated in the past where the focus was on the change from fully turbulent to laminar flows [15-17]. The reduction in Re was achieved using both suddenly expanding and diverging pipes so that change in Re was localized. The investigations were concerned with the evolution of spectral properties of the flow and exponential decay of turbulence was found with different exponents for central and near-wall regions. In our experiment, the chosen initial state of the flow was an equilibrium puff which was generated by introducing a short duration perturbation into Poiseuille flow. Then Re was reduced in a controlled way and the subsequent evolution of the puff was monitored as it progressed downstream.

A schematic diagram of a side-view of the apparatus is presented in Fig. 1. The pipe consisted of a tube of diameter $D=20 \pm 0.01 \mathrm{~mm}$ which was constructed using $150 \mathrm{~mm}$ long machined sections push fitted together and

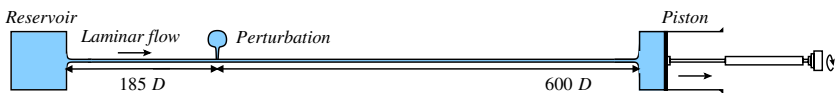

FIG. 1 (color online). Schematic diagram of the pipe flow facility (drawn to scale). The piston moves at a controlled speed and the fluid is pulled through the pipe at constant mass flux. The usable experimental run time is $\approx 30 \mathrm{~min}$. 
butted flush so that there was no measurable gap between each section. The pipe was held on a steel base with a total length of $15.7 \mathrm{~m}(785 \mathrm{D})$ and was aligned using a laser. A reservoir with a capacity of 100 liters was connected to the pipe through a smooth trumpet shaped inlet. A $30 \mathrm{~cm}$ diameter piston inside a ground steel cylinder pulled the fluid at a constant mass flux along the pipe using a computer controlled motor and lead screw arrangement. Hence, even if the fluid in the pipe became turbulent, the mass flux pulled through the pipe was unaffected, so that the Re remained constant. The experimental facility also allowed the speed of the piston to be varied as a function of time, i.e., the mass flux could be changed during a run to specified values in a controlled way. The long term temperature stability of the laboratory was controlled to $\pm 1{ }^{\circ} \mathrm{C}$ at a mean temperature of $20^{\circ} \mathrm{C}$. A typical temperature gradient recorded from several thermocouple along the pipe was $0.2^{\circ} \mathrm{C}$. By these means, we were able to maintain an accuracy in Re of better than $1 \%$. The facility enabled a laminar flow to be achieved up to a flow rate corresponding to $\operatorname{Re}=23000$. The flow state was monitored using Mearlmaid Pearlessence as flow visualization and single point velocity measurements were made using standard laser Doppler techniques in separate experiments.

An outline of the flow control procedure is shown in Fig. 2. Each experimental run proceeded as follows. An equilibrium puff was generated by introducing a perturbation into fully developed Poiseuille flow 185 diameters from the pipe entrance at $\mathrm{Re}=1900$. The perturbation was created using a boxcar pulse of fluid which was injected tangential to the main flow via a ring of six equally spaced $0.5 \mathrm{~mm}$ holes. The amplitude of perturbation was selected using the criterion established previously [9]. In principle, the perturbation will have a global effect on the flow but checks using push-pull disturbance generators shows that the perturbation is localized in practice. The puff was allowed to evolve for a further 100 diameters so that any of the known sensitivity to initial conditions [4] was lost. Then Re was reduced at a constant rate of $10 / \mathrm{sec}$ to a prescribed value in the range 1580-1740 and the evolution of the puff observed. The reduction in Re was achieved by decelerating the piston which pulled the flow under computer control so that the mass flux was reduced simultaneously all along the pipe. A settling time of $1 \mathrm{~h}$

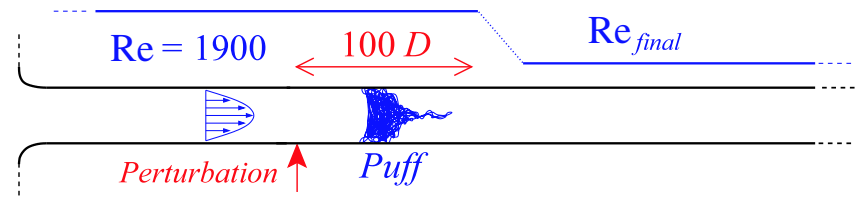

FIG. 2 (color online). Schematic of flow control procedure. Poiseuille flow was developed at $\mathrm{Re}=1900$ for $185 \mathrm{D}$ and a perturbation was injected (indicated by the arrow). The puff progressed downstream for $100 \mathrm{D}$ and $\mathrm{Re}$ was then reduced to a prescribed value. between each experimental run was found to be sufficient to ensure that disturbances in the header tank had decayed.

The initial effect of the reduction was that all of the puffs shrank to approximately half their original size during the deceleration phase. The weak wave structure at the head of the puff remained and there was evidence of vortical structures at the tail of the puff. The initial stages of decay were subtle but the final collapse was clear. In the latter stages, the block of vigorous disordered motion at the rear of the puff became detached from the wall and decayed rapidly. This was followed by the decay of the longer waves at the head of the puff. The distance taken for the disordered patch to decay was probabilistic in nature. Hence, the most useful measure which could be taken was the probability, $P$, of observing a localized disturbed region of flow as a function of distance downstream measured in pipe diameters $(D)$. The measurements were made using between 20 and 50 runs of the experiment for each value of Re. The results are shown in Fig. 3 where the point of injection of the initial perturbation corresponds to zero on the abscissa. The straight lines are least squares fits of exponentials $P(D) \propto \exp (-C D)$ to the experimental data points, where $C$ is a constant. Clear exponential decay is indicated by quality of the least squares fitted lines to the experimental points. It can be seen that the slopes increase as $\mathrm{Re}_{\text {final }}$ decreases, i.e., the distance required for the puff to decay is shorter for smaller $\mathrm{Re}_{\text {final }}$.

A measure which can be extracted from the exponential fits is the time required for half of the initial states to decay [18] and this is defined as the half-life $\tau=(\ln 2) / C$. A

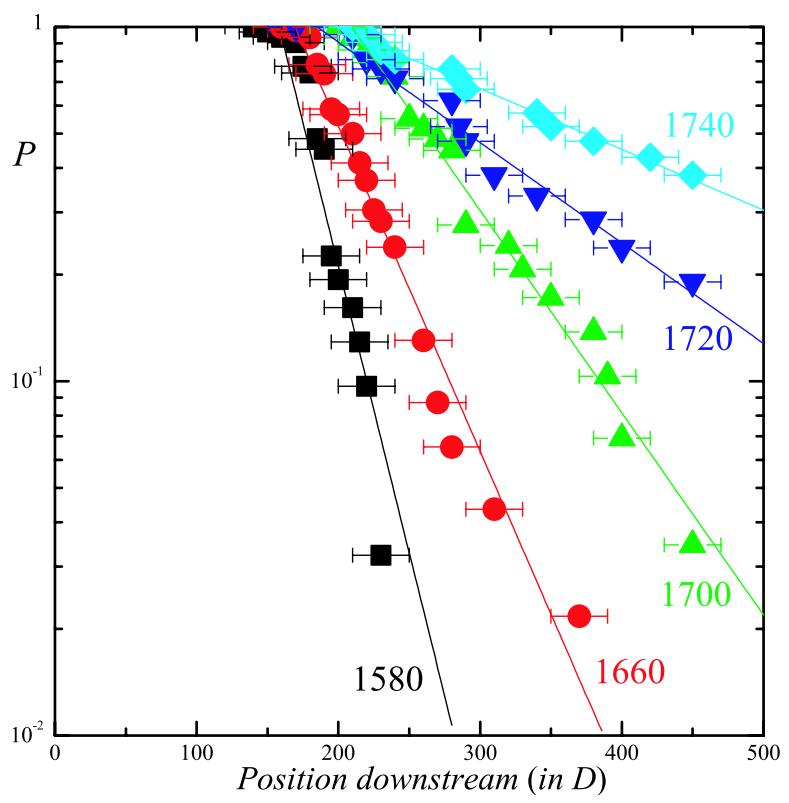

FIG. 3 (color online). Probability of observation of a puff versus downstream distance from the point of perturbation for a range of Reynolds numbers (1580-1740). The data are plotted on lin-log scales. The error bars indicate the experimental uncertainty of the measurements. 
graph of $\tau$ versus Re is shown in Fig. 4 where it can be seen that there is a sharp cutoff at $\operatorname{Re}_{c}=1750 \pm 10$, i.e., the half-life diverges at this value. More points have been used to construct Fig. 4 than available from the set of time histories represented in Fig. 3 (solid points). Some of the results (empty symbols) were obtained using different forms of perturbation including both injection and suction as well as combined injection and suction in one or several holes. In short, the results are independent of the method of creation of the puffs. Further tests on the robustness of the results included varying the distance that the puff was allowed to evolve between 10 and $500 \mathrm{D}$ and changing the deceleration rate of Re from 1 to $1000 / \mathrm{sec}$. The results were robust and were unaffected by any of these changes.

The exponential decay in the probability of observing a puff downstream suggests that, at each value of Re, they are independent events following a Poisson process. However, the least squares fit to the data in Fig. 4 shows that the half-life $\tau \propto\left(\operatorname{Re}_{c}-\operatorname{Re}\right)^{-1 \pm 0.02}$, which in dynamical systems theory is a generic feature associated with transient behavior where an attractor loses stability at a crisis $[5,6]$. In maps and low-dimensional dynamical systems the exponent has been found to be less than 1 but the qualitative features are similar to those uncovered here. Clearly describing the complicated state of turbulence with its associated spatiotemporal disorder using the language of low-dimensional strange attractors is fraught with difficulties [19]. However, if the puff travels at a well-defined speed it is perhaps justified to consider the temporal evolution of the system in a translating frame of reference while neglecting the spatial evolution. Moreover, at these

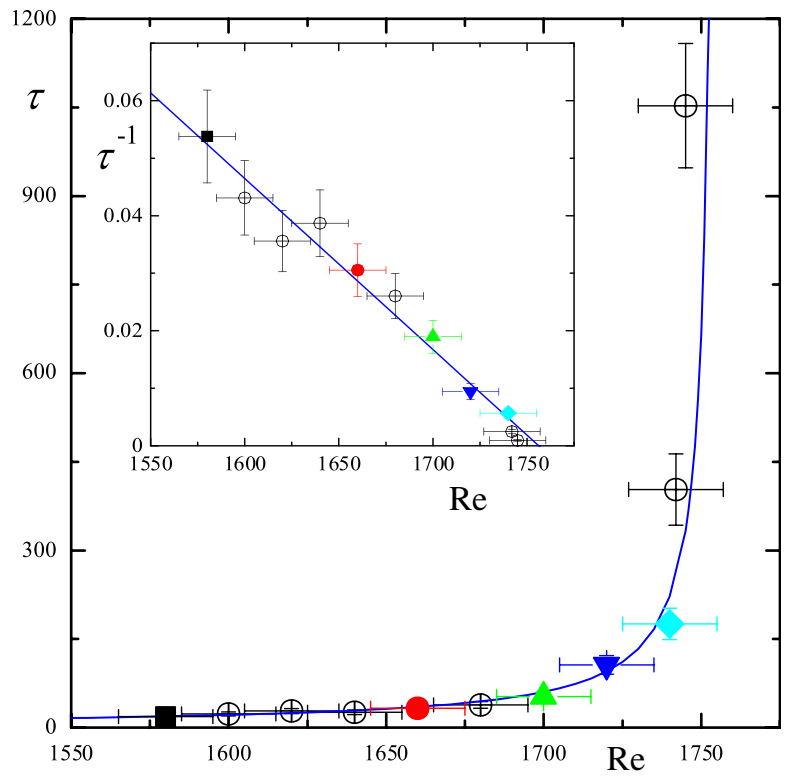

FIG. 4 (color online). Variation of the mean decay rate as a function of $\mathrm{Re}$ and a fit, which indicates a sharp cutoff at $\operatorname{Re}_{c} \approx$ $1750 \pm 10$. The inset is the inverse half-life versus $R e$ and a linear fit. relatively low values of $\mathrm{Re}$ it is well known that it contains some spatial and temporal order [11,14]. Therefore considering the puff as a translating low-dimensional complicated dynamical state is a reasonable first order approximation.

When $\operatorname{Re}$ is reduced to $1750 \pm 10, \tau \rightarrow \infty$, an interesting new type of behavior was observed. We illustrate this using the photographs and velocity-time traces shown in Fig. 5. The velocity-time series were measured using a laser Doppler velocimeter which measured the azimuthal flow component $0.2 D$ from the wall. A close-up view $(\approx 10 D)$ of the disordered region of an equilibrium puff $[4,11]$ at $\operatorname{Re}=1900$ is shown in Fig. 5(a). The entire puff is approximately 20 diameters long and we have omitted the decaying wavy structure which exists to the right of the field of view of the photograph. Small scale structures are evident and the puff reverts to laminar featureless flow abruptly in the left-hand side. The velocity trace is irregular although details of the temporal structure cannot be seen on the scale of Fig. 5(b). A flow visualization photograph of the state obtained after the reduction of $\operatorname{Re}$ to 1750 is shown in Fig. 5(c). The lifetime of the decaying disturbed flow approached infinity at this value of Re and hence the decay was very slow and the observed structure passed beyond the end of the pipe. Hence, it was not possible to decide whether this flow was stable or a long lived transient. The flow depicted in Figs. 5(c) and 5(d) is clearly more structured than those in Figs. 5(a) and 5(b) with a modulated wave pattern which has an axial wavelength of order of $1 D$. These structures were observed to emerge after the initial decay of the small scales but in this range of Re they had a more definite and regular form. In practice, the right-hand edge of this wavelike structure reverted to laminar flow via the weak decaying wave associated with puffs so that the total size of the wavy states was on the order of 50 pipe diameters.

It is interesting to note that wavelike structures are not a strong feature of experimental investigations of plane Couette flow [18,20-22], where exponential decay is also found. The initial conditions in plane Couette flow is a fully disordered patch of fluid which does not have the clear spatiotemporal structures found in an equilibrium puff. This important aspect is also a significant difference with numerical modeling work on Poiseuille flow where a very short calculation domain is used together with periodic boundary conditions [23] with fully disordered initial conditions. Another detail which can be seen in the flow visualization photograph in Fig. 5(c), is two longitudinal vortices at the left-hand side which shows a sharp contrast with the puff shown in Fig. 5(a). Moreover, the velocitytime trace shown in Fig. 5(d) contains an evident wave packet trace of wavelength $1.5 \mathrm{D}$. This is consistent with the global characteristic of the recently discovered development radial symmetric $(m=2)$ traveling wave which has a typical longitudinal wavelength of $1.5 D[7,8]$. 

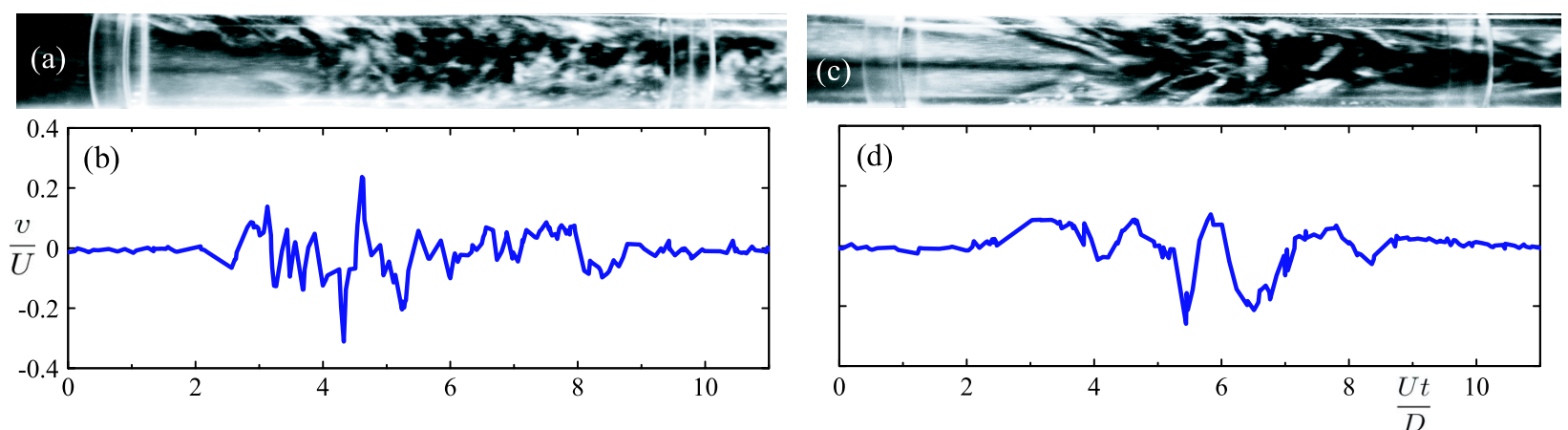

FIG. 5 (color online). Flow visualization (the flow is from left to right) and azimuthal velocity-time trace for initial and final flows: (a),(b) $\operatorname{Re}=1900$ and (c),(d) $\operatorname{Re}=1750$ after reduction of $\mathrm{Re}$. These wavelike states were observed in $10 \%$ of the experiments.

Our results demonstrate a clear threshold for laminarturbulent transition which is manifest in the approach to infinity of the lifetime of a disordered patch of fluid at a critical Reynolds number. Below the threshold, turbulence decays systematically via an exponential reduction in the probability of the propagation distance. Close to the threshold, the half-life becomes infinite and a new state which comprises a modulated wave packet emerges. An apparently simple scaling law with an exponent of one has been obtained by performing a "clean" experiment. The effects of initial conditions have been removed using a natural state of system, the equilibrium turbulent puff. Hence the effects of external noise, which can lead to long transients in dynamical systems [24] have been significantly reduced. The divergence in time scales provides a definite estimate with small error bars for the lower critical threshold which is in accord with previous values [13,17]. Perhaps, most importantly, our results indicate that the laminarization process progresses through structured stages and suggests a new research direction which will enable a detailed quantitative comparison between experiment and theory. This challenge may be met using a numerical approach with longer calculation domains where the spatiotemporal structure of the flow can be accommodated.

This research is supported by EPSRC via Grant No. GR/ 576137/01 (J. P.) and additional support (T. M.). We are grateful to R. R. Kerswell for helpful comments.

[1] O. Reynolds, Proc. R. Soc. Lond. A 34, 84 (1883).

[2] M. Fitzgerald, Phys. Today 57, No. 2, 21 (2004).

[3] P.G. Drazin and W.H. Reid, Hydrodynamic Stability (Cambridge University Press, Cambridge, England, 1980).
[4] A. G. Darbyshire and T. Mullin, J. Fluid Mech. 289, 83 (1995).

[5] C. Grebogi, E. Ott, and J. A. Yorke, Phys. Rev. Lett. 57, 1284 (1986).

[6] W. L. Ditto et al., Phys. Rev. Lett. 63, 923 (1989).

[7] H. Faisst and B. Eckhardt, Phys. Rev. Lett. 91, 224502 (2003).

[8] H. Wedin and R. R. Kerswell, J. Fluid Mech. 508, 333 (2004).

[9] B. Hof, A. Juel, and T. Mullin, Phys. Rev. Lett. 91, 244502 (2003).

[10] T. Mullin and J. Peixinho, in Proceedings of the IUTAM Symposium on Laminar-Turbulent Transition Bangalore, India, 2004, edited by R. Govindarajan and R. Narasimha (Springer, Bangalore, 2006), p. 45.

[11] I. J. Wygnanski and F. H. Champagne, J. Fluid Mech. 59, 281 (1973).

[12] G. Han, A. Tumin, and I. J. Wygnanski, J. Fluid Mech. 419, 1 (2000).

[13] D. A. Gilbrech and J. C. Hale, Developments in Mechanics (Pergamon, Case, 1965), Vol. II, p. 3.

[14] B. Hof et al., Science 305, 1594 (2004).

[15] K. R. Sreenivasan, Acta Mech. 44, 1 (1982).

[16] J. Laufer, in Mizellaneen der angewandten mechanik, edited by M. Schaefer (Academic-Verlag, Berlin, 1962), p. 166.

[17] M. Sibulkin, Phys. Fluids 5, 280 (1962).

[18] S. Bottin and H. Chaté, Eur. Phys. J. B 6, 143 (1998).

[19] S. H. Strogatz, Nonlinear Dynamics and Chaos (Perseus, New York, 1994).

[20] J. J. Hegseth, Phys. Rev. E 54, 4915 (1996).

[21] S. Bottin et al., Europhys. Lett. 43, 171 (1998).

[22] O. Dauchot and N. Vioujad, Eur. Phys. J. B 14, 377 (2000).

[23] H. Faisst and B. Eckhardt, J. Fluid Mech. 504, 343 (2004).

[24] Y. Do and Y.-C. Lai, Phys. Rev. E 71, 046208 (2005). 